\title{
Chapter 13 \\ Visualization of the Pathological Changes After Spinal Cord Injury (-From Bench to Bed Side-)
}

\author{
Masaya Nakamura
}

Spinal cord injury (SCI) results in devastating loss of function, because spinal cord of human beings never regenerates after injury. However, there is an emerging hope for regeneration-based therapy of the damaged spinal cord due to the progress of neuroscience and regenerative medicine including stem cell biology. Stimulated by the 2012 Nobel Prize in Physiology or Medicine awarded for Shinya Yamanaka and Sir John Gurdon, there is an increasing interest in the iPS cells and reprogramming technologies in medical science. There is no doubt that iPS cells are expected to open new era providing enormous opportunities in the biomedical sciences in terms of cell therapies for regenerative medicine.

We previously established a reproducible spinal cord injury model (SCI) in adult common marmosets and demonstrated that transplantation of human iPS derived neural stem/progenitor cells (NSPC) into the injured spinal cord promoted functional recovery (Kobayashi et al. 2012). There are several potential mechanisms of the functional recovery after iPS-NSPC transplantation including axonal regrowth, re-myelination and trophic support (Fig. 13.1). We confirmed these findings through the histological examinations such as immunostaining for GAP43, which is a marker for regenerating axons and Luxol fast blue (LFB) myelin sheath staining. The question is how to evaluate these findings in patients with SCI. We have to replace these histological examinations as imaging examinations in clinical setting. We sought to replace them as diffusion tensor tractography (DTT) and Myelin map. Furthermore, central re-organization could also occur after cell therapy and neurorehabilitation in SCI patients. In the previous animal study, we confirmed it with

Electronic Supplementary Material The online version of this chapter (https://doi. org/10.1007/978-981-13-7908-6_13) contains supplementary material, which is available to authorized users.

M. Nakamura $(\bowtie)$

Department of Orthopaedic Surgery, Keio University, School of Medicine,

Shinjuku, Tokyo, Japan

e-mail: masa@keio.jp 

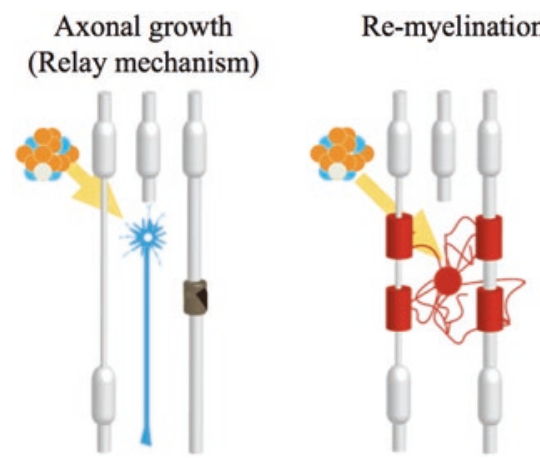

\section{Trophic support}

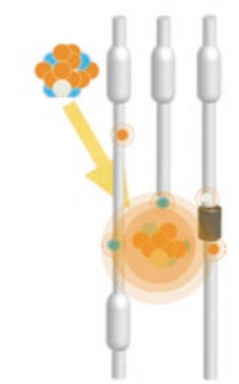

Fig. 13.1 Potential mechanisms of functional recovery after iPS-NSPC transplantation for SCI

tracer experiment to examine the changes in connectivity among the specific sites in brain. We also sought to replace them as connectivity analysis based on resting-state functional MRI.

\subsection{Diffusion Tensor Tractography}

\subsubsection{Basic Research}

Although the evaluation of axonal fibers is essential to assess the severity of SCI and efficacy of any treatment protocol, conventional methods such as tracer injection in brain parenchyma are highly invasive and technically demanding. Furthermore, since histological examinations are required to evaluate tracer studies, it has been impossible to evaluate axonal fibers in vivo and follow the sequential growth of axonal fibers in the same animal. We therefore sought to establish a non-invasive method to evaluate axonal fibers in vivo using DTT, a new MRI technique that makes in vivo tracing of axonal fibers possible. The properties and clinical applications of DTT in the brain have been reported, but technical difficulties have limited DTT studies of the spinal cord. Previously, we examined the effectiveness of DTT to visualize both intact and surgically disrupted spinal long tracts in adult common marmosets (Fujiyoshi et al. 2007). We induced a hemisection SCI at the 5th cervical vertebral (C5) level in adult common marmosets, and the spinal cord was imaged in a 7.0 Tesla MRI (Bruker Biospin) at several different time-points after injury. Furthermore, histological examination using Hematoxylin-Eosin, LFB and calmodulin dependent protein kinase to detect the corticospinal tract was conducted and confirmed the DTT findings in these animals.

We found that DTT clearly illustrated spinal projections such as the corticospinal tract and afferent fibers in control animals and depicted the severed long tracts in the injured animals. Furthermore, we succeed in demonstrating the pyramidal decussation of the marmoset. Histology of the spinal cords in both control and injured 
groups were consistent with DTT findings, verifying the accuracy of DTT. We also conducted DTT after contusion SCI in live marmosets and demonstrated that DTT can be performed in live animals to reveal in vivo nerve fiber tracing images, providing an essential tool to evaluate axonal conditions in the injured spinal cord (Konomi et al. 2012). Taken together, these findings demonstrate the feasibility of applying DTT to preclinical and clinical studies of spinal cord injury. DTT of the spinal cord is a powerful tool with tremendous potential if its properties and limitations are fully understood and correctly applied.

\subsubsection{Clinical Significance of DTT}

Based on our previous findings of DTT in adult common marmosets, we moved on to the study of DTT for cervical spondylotic myelopathy (CSM) to determine the clinical significance of DTT. The aims of this study were to (1) compare DTT of the cervical spinal cord in patients suffering from CSM with their clinical assessment, (2) compare DTT with the signal intensity of the spinal cord achieved with conventional MRI sequences and (3) determine whether postoperative changes in DTT have prognostic significance of patients with CSM after decompression surgery (Nakamura et al. 2012).

In twenty-one patients with CSM, T2-weighted imaging and DTI were performed before and 1 year after the decompression surgery. The value of fractional anisotropy was analyzed and fiber tracking was performed and analyzed using VOLUME-ONE software. Fiber tracts were generated by placing the region of interest (ROI) at the second cervical vertebral (C2) level (normal spinal cord) or at the most compressed level with reference to the sagittal MR images. The tract fiber (TF) ratio was calculated using a following formula: (Number of the tract fibers at the most compressed level) / (Number of the tract fibers at C2 level) $\times 100 \%$. Japanese Orthopaedic association (JOA) scoring system of CSM was used to determine pre- and postoperative functional status and recovery rate was calculated by Hirabayashi method. We performed statistical analyses to determine the differences in preoperative JOA score, preoperative TF ratio and recovery rate between the patients with and without high signal intensity on T2-weighted image: T2HSI (+) and (-) groups. We also performed Nonparametric correlation analysis to determine the relationship between pre- and post-operative JOA scores and pre- and postoperative TF ratio respectively and also preoperative TF ratio and recovery rate.

We found that there was a significant difference in preoperative JOA scores between T2HSI (+) and (-) groups, whereas no significant difference in the recovery ratio was observed between them. There was also no significant correlation between the preoperative JOA score and the preoperative TF ratio, suggesting that the preoperative TF ratio did not reflect the severity of neurological deficit before the surgery. In contrast, there was a significant correlation between the preoperative $\mathrm{TF}$ ratio and the recovery rate. It was noteworthy that we can anticipate a poor prognosis (recovery ratio $<40 \%$ ) of patients with CSM, whose preoperative TF ratio is 
Fig. 13.2 Clinical application of DTT to Cervical Spondylotic Myelopathy A poor outcome (recovery rate $<40 \%$ ) could be anticipated for CSM patients with preoperative tract fiber ratios below $60 \%$

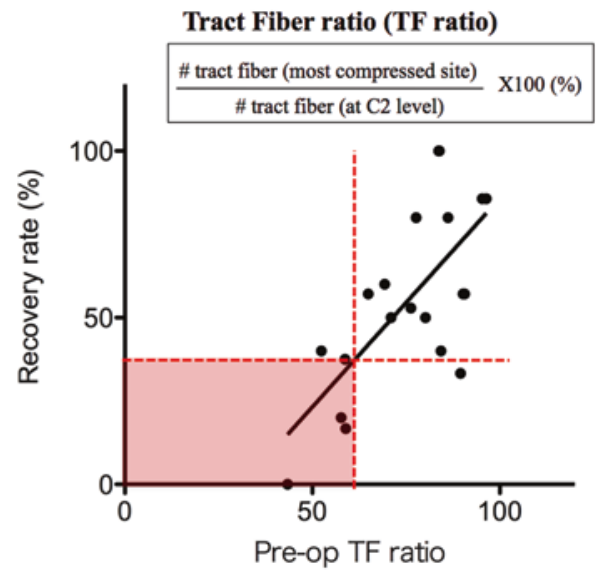

below $60 \%$ (Fig. 13.2). Taken together, preoperative TF ratio was significantly correlated with CSM patients' recovery rate, suggesting that preoperative DTT could become a new prognostic predictor of CSM patient after decompression surgery.

\subsection{Myelin Map}

\subsubsection{Basic Research}

To assess the severity of SCI and efficacy of its treatment, it is essential to evaluate the histopathological state of myelin sheaths in the injured spinal cord. Therefore, the development of a new method for non-invasively evaluating myelin sheath was desired. We developed a novel technique, called the Myelin map, which uses pulsed gradient spin-echo MRI to visualize myelin in vivo, by focusing on the non-Gaussian diffusion distribution of water molecules confined by myelin sheaths (Fujiyoshi et al. 2016).

First, to validate the accuracy of this technique in vivo, we obtained Myelin maps of the spinal cords of postmortem myelin-deficient shiverer and jimpy mice as well as wild-type littermates, and compared them with histological findings of the same tissues. We found that there were almost no myelin sheathes in the spinal cords of myelin-deficient mice, which were closely associated with the findings of Myelin map, but not the other images such as T2-weighted image and FA map.

Second, we induced chemical SCI by injecting lysophosphatidylcholine into the posterior funiculus as well as contusive SCI at the C5 level using a modified NYU device in adult common marmosets. Furthermore, we performed transplantation of NSPC into the contused spinal cord of adult common marmosets. We took myelin map using a 7.0 Tesla MRI at the several different time-points. Myelin maps accurately depicted the demyelination/remyelination in marmoset chemical SCI, as well 
as the remyelination after allogeneic NS/PC transplantation in common marmosets. Immuno-EM analysis revealed that the transplanted NSPCs actively re-myelinated the de-myelinated axons in the injured spinal cords of common marmosets.

Taken together, we succeeded in developing a straightforward way to image the myelination of the spinal cord that should allow clinicians to evaluate the normal and pathological state of myelination more easily than with current methods. Using Myelin map, we also succeeded in obtaining strong evidence that the remyelination brought by transplanted NSPCs is a key mechanism for functional recovery. Myelin maps have an unparalleled ability to non-invasively visualize both normal myelin and its pathological state, and therefore this technique promises to be a powerful tool for researchers and clinicians examining diseases of the central nervous system. Furthermore, this method uses equipment that is already widely available, and so has the potential for immediate clinical application.

\subsubsection{Clinical Significance of Myelin Map}

Based on the findings of our basic research about Myelin map, we moved onto the clinical application of Myelin map. Brain MRI of neuromyelitis opticaspectrum disorders (NMOSD) patients often reveals multiple T2 high signal lesions, for which patients may be misdiagnosed as multiple sclerosis (MS) and treated with MSmodifying drugs potentially harmful to NMOSD. It is well known that distinct pathologies exist in NMOSD and MS, however the correct diagnosis is often jeopardized by the non specific nature of T2 signals. We sought to determine whether Myelin map enable us to differentiate NMOSD from MS.

A 54yearold female patient with newly diagnosed NMOSD with positive antiaquaporin 4 antibody serostatus and a 30yearold female patient with 7month history of relapsingremitting MS were included in the study. Both patients experienced acute exacerbation explainable by Gadolinium (Gd)enhancing T2 lesion and they were treated with intravenous methylprednisolone therapy (IVMP). 3 T MRI studies with conventional modalities (T1 and T2weighted images, with Gd enhancement) and myelin map were repeated before and after IVMP with 3month intervals (Tanikawa et al. 2017).

In both NMOSD and MS patients, T2 lesions surrounded Gdenhancing inflammatory rims, however Myelin map supported demyelination existed outside the rim in NMOSD whereas it was localized inside the rim in MS. After the IVMP, Gdenhancing rims disappeared in both cases, albeit T1 hypointense cavity was left behind only in NMOSD. T2 lesion remained around the cavity in NMOSD or inside the diminished rim in MS, but neither of them were indicative of persistent demyelination. Myelin map supported remyelination was fast and extensive, already near complete at 3 months post IVMP in NMOSD, whereas remyelination slowly progressed inward from the rim, but remained incomplete even at 6 months postIVMP in MS. In conclusions, Non-myelin specific nature of T2 signals were confirmed in both NMOSD and MS. Distinct patterns in relative location of 
demyelination to inflammatory core may suggest differential pathogenesis underlying these diseases. Our cases also suggest a possible difference in remyelination capacity in NMOSD and MS, however a larger clinical study is required to draw a final conclusion.

\subsection{Resting-State Functional MRI}

It is recently reported that after SCI, neuronal connectivity changes occur not only in the spinal cord, but also in the brain. However, there has been no report investigating the changes of the neuronal functional communication among the several regions of the cerebral cortex after SCI.Therefore, we examined the changes in neuronal functional connectivity of the brain after SCI by taking the resting statefunctional MRI (rs-fMRI) (Matsubayashi et al. 2018).

Adult mice were subjected to rs-fMRI without anesthesia. After careful acclimation to environmental stress in taking MRI, rs-fMRI was performed and the data of different mice brain were standardized with the stereotaxic MRI brain template. By classifying the regions of the brain based on the Allen mouse brain atlas, the neuronal functional connectivity was analyzed among the specific regions. Next, complete transection or contusion SCI was induced at the 10th thoracic vertebral level in these mice. rs-fMRI was taken at the several time-points after injury and the changes in neuronal functional connectivity of their brain were visualized using SPM12 software and CONN toolbox, and these data were analyzed using graph theory.

We succeeded in detecting the normal neuronal functional connectivity in the brain of the awake mice through rs-fMRI. In the comparative analyses before and after the transection SCI, the changes in functional connectivity was observed between the primary and secondary motor cortex. Moreover, by analyzing the brain connectivity after the contusion SCI, we detected the changes in the neuronal functional connectivity in accordance with the motor function recovery. Based on the graph theory, quantitative analyses of the whole brain structural community revealed the decrease in the density of the whole brain network and the changes in the pattern of the components of the specific brain community structure after SCI. In the current study, we demonstrated the feasibility to examine neuronal functional connectivity in the brain of awake mice using rs-fMRI. We also showed the relative changes in the neuronal functional connectivity in the brain after SCI and identified the regions that were strongly related to the functional recovery after SCI. In addition, we detected that the networks changed in the brain after SCI by the analyses using a graph theory. These networks were divided into small communities and the density of these networks decreases immediately after SCI, then individual regions change the importance of their network, suggesting that brain networks were reorganized and changed the efficiency of the entire network after SCI. 


\section{References}

Fujiyoshi K, Hikishima K, Nakahara J, Tsuji O, Hata J, Konomi T, Nagai T, Shibata S, Kaneko S, Iwanami A, Momoshima S, Takahashi S, Jinzaki M, Suzuki N, Toyama Y, Nakamura M, Okano $\mathrm{H}$ (2016) Application of q-space diffusion MRI for the visualization of white matter. J Neurosci 36(9):2796-2808

Fujiyoshi K, Yamada M, Nakamura M, Yamane J, Katoh H, Kitamura K, Kawai K, Okada S, Momoshima S, ToyamaY, Okano H (2007) In vivo tracing of neural tracts in the intact and injured spinal cord of marmosets by diffusion tensor tractography. J Neurosci 27(44):11991-11998

Kobayashi Y, Okada Y, Itakura G, Iwai H, Nishimura S, Yasuda A, Nori S, Hikishima K, Konomi T, Fujiyoshi K, Tsuji O, Toyama Y, Yamanaka S, Nakamura M, Okano H (2012) Pre-evaluated safe human iPSC-derived neural stem cells promote functional recovery after spinal cord injury in common marmoset without tumorigenicity. PLoS One 7(12):e52787

Konomi T, Fujiyoshi K, Hikishima K, Komaki Y, Tsuji O, Okano HJ, Toyama Y, Okano H, Nakamura M (2012) Conditions for quantitative evaluation of injured spinal cord by in vivo diffusion tensor imaging and tractography: preclinical longitudinal study in common marmosets. NeuroImage 63(4):1841-1853

Matsubayashi K, Nagoshi N, Komaki Y, Kojima K, Shinozaki M, Tsuji O, Iwanami A, Ishihara R, Takata N, Matsumoto M, Mimura M, Okano H, Nakamura M (2018) Assessing cortical plasticity after spinal cord injury by using resting-state functional magnetic resonance imaging in awake adult mice. Sci Rep 8(1):14406

Nakamura M, Fujiyoshi K, Tsuji O, Konomi T, Hosogane N, Watanabe K, Tsuji T, Ishii K, Momoshima S, Toyama Y, Chiba K, Matsumoto M (2012) Clinical significance of diffusion tensor tractography as a predictor of functional recovery after laminoplasty in patients with cervical compressive myelopathy. J Neurosurg Spine 17(2):147-152

Tanikawa M, Nakahara J, Hata J, Suzuki S, Fujiyoshi K, Fujiwara H, Momoshima S, Jinzaki M, Nakamura M, Okano H, Takahashi S, Suzuki N (2017) q-Space Myelin Map imaging for longitudinal analysis of demyelination and remyelination in multiple sclerosis patients treated with fingolimod: a preliminary study. J Neurol Sci 373:352-357

Open Access This chapter is licensed under the terms of the Creative Commons Attribution 4.0 International License (http://creativecommons.org/licenses/by/4.0/), which permits use, sharing, adaptation, distribution and reproduction in any medium or format, as long as you give appropriate credit to the original author(s) and the source, provide a link to the Creative Commons licence and indicate if changes were made.

The images or other third party material in this chapter are included in the chapter's Creative Commons licence, unless indicated otherwise in a credit line to the material. If material is not included in the chapter's Creative Commons licence and your intended use is not permitted by statutory regulation or exceeds the permitted use, you will need to obtain permission directly from the copyright holder.

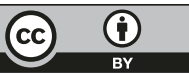

